\title{
Axially symmetric large-scale transport of the magnetic flux during solar activity cycle
}

\author{
Pavel Ambrož \\ Astronomical Institute of the Academy of Sciences of the Czech Republic, \\ CZ-25165 Ondřejov, Czech Republic \\ email: pambroz@asu.cas.cz
}

\begin{abstract}
Transport of the solar background large-scale magnetic regions is followed between individual consecutive magnetic synoptic charts derived from observing data of Kitt Peak NSO. During many solar rotations the horizontal magnetic flux displacement was described by largescale horizontal transport velocities, inferred in many points over the whole solar photosphere. Large-scale transport velocities contains from both axially symmetric and non-axially symmetric components. The first one describes zonal and meridional global transport studied in time interval during three last solar activity cycles. Cycle dependent global velocities are found as values varying in heliographic latitude and in the phase of the solar cycle.
\end{abstract}

Keywords. Sun: magnetic fields, rotation, convection

Distribution of solar activity and of magnetic fields indicates that also the non-axially symmetric processes play an important role on the Sun. In our investigations we suppose that magnetized plasma is horizontally transported mainly by large-scale flow (Ambrož, 2001). Recently similar velocity structures were obtained by Zhao and Kosovichev (2004), on the basis of measuring methods of solar helioseismology. The Local Correlation Tracking (LCT) method derived by November (1986) gives the possibility to determine horizontal velocities in all points on the solar surface. The LCT method is suitable in case of smooth input data and therefore it is necessary to use the smoothed magnetic fields.

The latitude profiles of the zonal velocities are mounted in the chronological order into the time-latitude diagram. The dominant result is that the mean values of zonal velocity are dependent on the 11 year activity cycle. The positive zonal velocity (orientated in the sense of the rotation) is maximum on the equator and the velocity cycle maximum values were detected about one year before the activity cycle minimum.

The meridional velocity is inferred from the meridional displacement of parts of the magnetic regions. The meridional profiles for each individual Carrington rotation (CR) provide cycle dependent patterns of symmetry with the solar equator. Around the minimum phase of the activity cycle, the meridional velocity is shortly (about $10 \mathrm{CRs}$ ) oriented chaotically. The main phase with pole-ward flow in low latitudes and equator-ward flow in higher latitudes is observed and this regime covers nearly whole solar activity cycle. The amplitude of the mean meridional velocity is from 10 to $15 \mathrm{~ms}^{-1}$.

Distribution of zones with north-ward and south-ward oriented both mean velocity vectors is, however, not identical for different amount of averaging of the local meridional velocities. The averaging of both zonal and meridional transport velocities is influencing as a filter, emphasized mean transport velocities in regions with different characteristic dimension.

Inferred values of the large-scale horizontal velocity fields are available nearly for three solar activity cycles (Nos. 21-23). Data covers together 368 Carrington synoptic charts, each with sampling interval $1^{\circ}$ in both heliographic longitude and latitude and temporal 


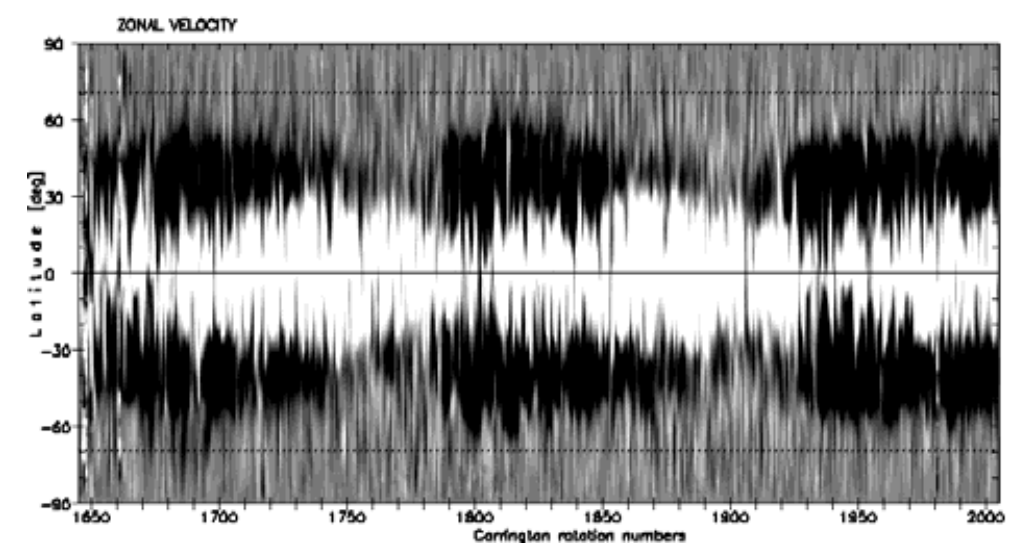

Figure 1. Time-latitude diagram of the zonal velocities. Velocities in dark/bright regions are oriented again/in sense of solar rotation.

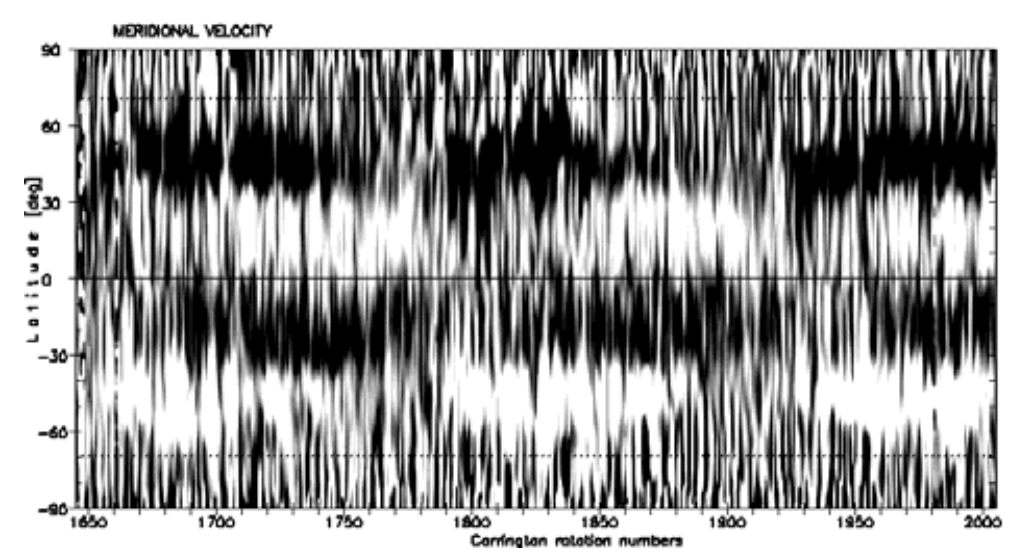

Figure 2. Time-latitude diagram of the meridional velocities. Velocities in dark/bright regions are oriented to south/north poles.

resolution 1 Carrington rotation period. The patterns derived from all velocity components varies in relationship with 11 year activity cycle, but also the variations with shorter periods are observed. For all components the structures are equatorially symmetric, but the symmetry is neither simple nor perfect. The axially symmetric concept of the solar dynamo seems to be a simplified mechanism of the much more complex process, where the large-scale flow plays an important role.

\section{Acknowledgements}

Present work was supported by grant of GA/AVČR No. A300030506 and AV0Z10030501. NSO/Kitt Peak data used in the present paper are produced cooperatively by NSF/NOAO, NASA/GSFC, and NOAA/SEL.

\section{References}

Ambrož, P. 2001a, Solar Phys. 198, 253

November, L. J. 1986, Applied Optics 25, 392

Zhao, J. and Kosovichev, A. G. 2004, Astrophys. J. 603, 776 